\title{
Aportaciones de la tecnología a la e-Evaluación
}

\section{Technological contributions to e-Assessment}

\author{
Elena Barberà \\ Estudios de Psicología y Ciencias de la Educación \\ Universidad Oberta de Catalunya. España. \\ ebarbera@uoc.edu
}

\begin{abstract}
Resumen
En el presente artículo se expone un concepto complejo de evaluación de los aprendizajes que incluye cuatro dimensiones que se consideran básicas para una práctica evaluativa completa: evaluación del aprendizaje, evaluación para el aprendizaje, evaluación como aprendizaje y evaluación desde el aprendizaje. Se propone recuperar esta perspectiva compleja en la evaluación de los aprendizajes en los contextos virtuales mediante el análisis de las aportaciones específicas que introduce la tecnología en este campo. De esta manera se distinguen como contribuciones particulares de la tecnología en estos contextos en cuanto modifican significativamente las prácticas presenciales que conocemos: la evaluación automática, la evaluación enciclopédica y la evaluación colaborativa. El artículo acaba resaltando el valor de los procesos comunicativos de los resultados a los alumnos $\mathrm{y}$, en concreto, el valor de la retroalimentación y la necesidad y posibilidades que ofrece la tecnología para su aprovechamiento.
\end{abstract}

\section{Palabras clave}

Evaluación del aprendizaje, evaluación formativa, contexto virtual, retroalimentación, evaluación y tecnología.

\begin{abstract}
The article explains a complex concept about assessment of learning in virtual contexts. It includes four dimensions: assessment of learning, assessment for learning, assessment as learning and assessment from learning.

The main contributions of technology in that field complementary to face-to-face practices are developed. In this way, the article presents three type of significant assessments: automatic assessment, encyclopedic assessment and collaborative assessment. The paper ends underlining the value of the post evaluative practices like assessment results communication, virtual feedback and the contribution of the technology for the exploitation of the feedback process..
\end{abstract}

\section{Keywords}

Assessment of learning, formative assessment, virtual context, feedback, assessment and technology

\section{Introducción}

¿Realiza la tecnología verdaderamente aportaciones específicas en el campo de la evaluación de los aprendizajes o se trata de una mera copia de la evaluación que se realiza en las aulas presenciales? Si las hubiere, estas aportaciones ¿facilitan o entorpecen el proceso de enseñanza y aprendizaje o simplemente se trata de un formato distinto y acorde al contexto virtual de educación? 
Estas y otras preguntas conforman el motivo de este artículo que simplemente quiere aportar elementos para la discusión y reflexión conjunta sobre el complejo tema de la evaluación que se realiza de manera remota con presencia de la tecnología en diferentes ámbitos educativos.

Para ello, en primer lugar, en este artículo se inicia la exposición, a modo de introducción, con la valoración global de la evaluación del aprendizaje en los contextos virtuales. Se apuntan algunos puntos fuertes y puntos débiles de la comunicación y de la enseñanza en red (Mäkitalo, Salo, Häkkinen y Järvelä, 2001) para después pasar a describir algunos de los efectos que la evaluación tiene sobre el aprendizaje.

Esta primera parte va seguida de una segunda que se refiere al propio concepto de evaluación adaptable a los contextos virtuales. Como diseñadores, docentes o directivos se tiene la oportunidad de influir o cambiar la enseñanza pero parece que nos empeñamos en reproducir viejos esquemas en estos nuevos contextos. Como veremos y como creemos, la evaluación es una herramienta de innovación imprescindible para que se de este cambio en pro de un aprendizaje más significativo para los alumnos, pero en muchos casos nos encontramos que utilizamos la tecnología para realizar una mera copia de lo que se realiza en los contextos presenciales.

El tercer apartado se dirige a responder preliminarmente las preguntas que nos hacíamos al principio sobre las aportaciones que la tecnología hace a la evaluación. Es decir ¿Qué aporta realmente la tecnología?, ¿Hay algún cambio realmente significativo?, ¿Cuáles son?, ¿Qué sentido tienen?

Después de tratar estas aportaciones nos introducimos en un cuarto apartado en el que abordaremos algunos focos que no se pueden eludir en el momento de pensar en innovar en educación en línea, siempre situados en el marco de no renunciar a un aprendizaje más constructivo y no tan reproductivo o asociativo. Acabaremos con una agenda en la que hablaremos de esos focos de innovación para la evaluación. Si enfoques evaluativos son importantes en toda acción formativa de ámbito formal parece que en los sistemas virtuales este hecho cobra una mayor relevancia puesto que en muchos de ellos el proceso formativo está basado en una evaluación continua o en la continua propuesta de tareas. Así, en la enseñanza virtual el eje vertebrador en muchos casos son las actividades y tareas en el marco de una evaluación continua que se realiza con soporte de la tecnología.

\section{Valoración de la práctica educativa virtual}

En una rápida valoración, quizá un poco interesada pero no falaz -puesto que entendemos que existen otras problemáticas más globales (Barron, 2004)-, algunos de los puntos débiles y que puntos fuertes de la enseñanza superior en línea desde el punto de vista de los alumnos serían los que exponen a continuación. Así, uno de los puntos fuertes es la flexibilidad horaria y espacial que asume el tópico de 24x7x365 (24 horas al día, por siete días de la semana, por 365 días del año) de atención al alumno. Esta flexibilidad horaria queda en principio asegurada cuando la comunicación es asincrónica. También se considera una ventaja la información que se aporta al alumno en línea sobre la totalidad de la secuencia didáctica que seguirá en un periodo de tiempo concreto y necesariamente programado. El alumno tiene que contar con una perspectiva 
de todo el proceso formativo de un modo claro que va más allá de la clase a la que asiste en un momento determinado como puede pasar en muchos casos en la enseñanza presencial. Por tanto tiene un plan de trabajo, unas guías de estudio y unos calendarios que le orientan en esta secuencia temporal de aprendizaje. Otro de los puntos fuertes es, sin duda, el mismo marco en el que se enclava el proceso de enseñanza y aprendizaje: el ciberespacio, su acceso a él y todo lo que supone a nivel comunicativo e informativo. No profundizaremos en ese tema desde esta perspectiva, pero es una ganancia clara por parte de los alumnos como lo es el primer punto citado sobre la posibilidad que tienen de consultar de manera abierta en estos contextos virtuales de enseñanza-aprendizaje.

Trasladándonos a los puntos débiles se podría señalar, en primer lugar y en contraposición de lo apuntado en el apartado de ventajas sobre la flexibilidad horaria y espacial de acceso que existe una cierta inflexibilidad instruccional, en tanto que al final la docencia se convierte en un cúmulo de tareas con fechas de finalización e inicio. Es decir, la posibilidad de acceso y consulta es abierta pero al final el proceso instruccional se resume en una serie de trabajos parcelados y con pocas relaciones internas entre ellos disminuyendo una consecución más dúctil y adaptada desde la perspectiva del aprendiz y del propio aprendizaje. El alumno espera más personalización y una ejecución de tareas más progresiva pero la enseñanza, en este punto, se manifiesta, en términos generales, bastante rígida (Anta, 2002). Otro punto que se puede resaltar es el retorno cualitativo que se da a los trabajos realizados en línea en cuanto al ajuste que realiza el profesor $\mathrm{u}$ otros alumnos sobre el contenido de aprendizaje. Este aspecto aunque muestra mucho potencial de cambio es un punto de los más débiles que hay junto con el desarrollo de trabajo en entornos colaborativos. De manera relacionada, otro punto débil son también los criterios de evaluación y de comunicación de resultados que trataremos más adelante; asimismo la interacción del profesor y el alumno que tienen sobre el contenido es otro aspecto que muestran una alta fragilidad en los contextos virtuales. ¿Qué sucede en los contextos virtuales asincrónicos de enseñanza-aprendizaje que se escribe mucho, profesores y alumnos, sobre la manera de proceder -dónde enviar una tarea, qué formato utilizar, cómo realizar el debate, etc.- pero no tanto sobre el contenido específico del área y el ámbito de conocimiento que es lo que al final interesa más? Otro punto interesante que se valora como un aspecto débil es la propia sensación de logro que tiene el alumno; el alumno va ejecutando tareas y teniendo resultados de ellas pero consigue con cierta dificultad una idea de conjunto de la materia y de su progresión en ella.

Si revisamos los puntos débiles y los puntos fuertes que se han apuntado una posible conclusión es que los aspectos de carácter más robusto se refieren a puntos aplicados a la planificación y estructuración de la enseñanza y el aprendizaje y en los aspectos de carácter más endeble encontramos muchos puntos relativos a la evaluación de los aprendizajes. Aceptado lo anterior se puede concluir que la evaluación del aprendizaje es algo pendiente de mejorar, algo sobre lo que hay que reflexionar con mayor profundidad, que hay que desarrollar de un modo más creativo y volver sobre ello invirtiendo más recursos pedagógicos y tecnológicos con el fin no sólo de innovar sino de llegar a una evaluación satisfactoria para profesores, alumnos e instituciones. 


\section{Influencias de la evaluación}

Un breve repaso a algunas de las influencias y de los efectos más notables que la evaluación misma tiene sobre el proceso de enseñanza-aprendizaje ayudará a calcular la relevancia de la evaluación para el aprendizaje (Barberà, 2003). Estas influencias son de diferente cariz y tienen un peso evidente pero no siempre visible en el momento de evaluar a los alumnos en un contexto formal de educación. La primera influencia, que se reconoce con claridad, es la influencia de tipo motivacional y se refiere a la tensión que se reconoce en los alumnos en tanto en cuanto sus resultados tienen una repercusión social. Es decir, cuando a un alumno se le comenta que va a ser evaluado, por este simple hecho, el alumno despliega un dispositivo de alerta que normalmente ligado a una mayor atención o, mejor dicho, interés sobre las tareas que se realizan. Esa influencia que consideramos como motivación externa sería deseable poderla reconvertir en un fenómeno más intrínseco.

La segunda influencia es la que se refiere a una influencia de consolidación. Es importante entender la evaluación como un momento particular de enseñanzaaprendizaje y no sólo como algo que se sitúa, al final o en medio del proceso formativo mismo, sino que también tiene el efecto de afianzar el contenido que se está tratando o de "acabar de aprender". Es importante entonces que cuando se evalúe, se reconozca este proceso de afianzamiento o se de relevancia al contenido que se está desarrollando y no se haga una selección de contenidos al azar. No se trata de formular preguntas casuales o aquellas que nos vienen a la cabeza en un momento determinado sino que es necesaria una planificación de competencias evaluadas que se siguen o acaban de consolidar en el momento mismo de la evaluación.

La última influencia que destacamos es la influencia de carácter anticipatorio de la evaluación sobre el proceso de enseñanza y aprendizaje: la evaluación ejerce el poder de modelar el proceso de aprendizaje aportando a los alumnos directrices claras de cómo actuar en este contexto. El primer día de clase al exponer el plan docente los alumnos están muy interesados en saber cómo van a ser evaluados. En el contenido de esta información ellos están captando y actualizando cómo serán los requisitos de la evaluación y a qué premisas responderá. Este conocimiento les servirá después, a lo largo de su proceso de estudio de la materia, para adaptar su manera de estudiar e incluso de aprender. El ejemplo más ilustrativo lo tenemos en que si evaluamos a los alumnos con exámenes tipo test ellos muy probablemente memorizaran los contenidos de una manera diferente de si se les propone actividades de elaboración o de construcción. En resumen, estas influencias que aquí se plantean llevan a valorar la evaluación como un momento especial de influencia e impacto para los alumnos, por lo menos, estos tres motivos que se han expuesto con anterioridad.

\section{Concepto multidimensional sobre la evaluación}

Seguimos con el concepto de evaluación porque es importante compartir lo que significa en toda su complejidad el hecho de evaluar. En términos generales se puede decir que la evaluación se reconoce pero no se conoce. En un primer momento parece que no hay dudas sobre lo que es o no es evaluación en un contexto educativo formal pero no acertamos a destacar los aspectos clave en su conjunto, por lo menos, de un modo equilibrado. 
La evaluación puede ser entendida como evaluación del aprendizaje, es decir, la evaluación que nos da como resultado -fruto de la aplicación de la función más normativa y social de la evaluación- la conformidad de si los alumnos son o no son capaces delante de la sociedad de saber y de ser competentes en un determinado ámbito. Si bien esta parte no es en absoluto banal se considera que la evaluación va más allá de este hecho acreditativo.

Así, la evaluación no es solamente evaluación del aprendizaje sino que es también evaluación para el aprendizaje. En la evaluación para el aprendizaje el eje motor principal es la retroalimentación y el aprovechamiento que de ésta realizan los alumnos y los mismos profesores. Es en el marco del diálogo entre profesor y alumnos que se organiza alrededor del contenido y del quehacer académico en el que se ofrece una ayuda y respuesta ajustada, coherente, y contextualizada en la materia de estudio que sirve para avanzar en el conocimiento.

Pero no sólo se han de contemplar las dos dimensiones citadas de evaluación del y para el aprendizaje. También se debería añadir la evaluación como aprendizaje. Esta dimensión contempla el aprendizaje mismo de la dinámica evaluativa en cuanto análisis y reflexión de las propias prácticas educativas llevadas a cabo por los propios alumnos. Esta reflexión aprendida conlleva la posibilidad de regular el propio aprendizaje adaptándolo a los fines educativos y a los intereses personales. La dinámica reflexiva en el marco de la evaluación es un hecho singular que sólo la facilitan algunas opciones metodológicas de evaluación (por ejemplo, evaluación por portfolios).

Por último la cuarta dimensión, que no queremos olvidar y que quizá tendríamos que haber empezado por ella, es la evaluación desde el aprendizaje. Es comúnmente aceptada la idea de que aprender es conectar el conocimiento nuevo al que accedemos por primera vez con el conocimiento que ya poseemos. Por tanto, esta conexión se tiene que desarrollar de manera significativa por lo que es condición necesaria conocer el punto de partida de ese conocimiento, es decir, lo que se sabe previamente al acto educativo. De este modo, los conocimientos previos y el sentido con el que accede el alumno a los aprendizajes devienen elementos esenciales para la docencia pudiendo desde allí anclar y desarrollar lo que se enseña a los alumnos. Estas cuatro dimensiones entrelazadas en la práctica evaluativa quizá no aseguran un aprendizaje sin errores pero facilitan sobremanera el cambio educativo en los contextos formales de enseñanza y aprendizaje.

En este marco multidimensional para acabar con esta primera fase genérica sobre el hecho evaluativo y después de valorar los puntos débiles y puntos fuertes de la evaluación, nos adentramos en el foco del artículo que pretende aproximarse, aunque no totalmente, a la respuesta de la pregunta de cuáles son las aportaciones de la tecnología a la evaluación formal sobre los aprendizajes que se realizan en línea.

\section{Aportaciones de las TIC}

Sin entrar a valorar el marco educativo modificado a causa de la introducción de la tecnología se resaltan tres grandes cambios que la tecnología ha aportado en el contexto de la evaluación. Con riesgo de inexactitud en la clasificación de los hechos, 
identificamos cada aportación mediante una etiqueta que busca caracterizar el tipo de aportación realizado por la tecnología. En síntesis, se trata de: 1. La evaluación automática, en el sentido que la tecnología contiene bancos de datos que se relacionan entre ellos y se pueden ofrecer a los alumnos respuestas y correcciones inmediatas. Las pruebas electrónicas tipo test que incluyen respuestas correctas ejemplifican este tipo de aportación. 2. La segunda aportación de la tecnología en el campo evaluativo se identifica mediante una evaluación de tipo más enciclopédico, en referencia al cúmulo de contenidos que se manejan de una fuente más compleja o de diferentes fuentes. En este caso la ejemplificación más clara se refiere a la elaboración de trabajos monográficos de ensayo sobre una temática específica y para el que se cuenta con Internet como un repositorio de información excepcional y muy completa. 3. Y la tercera aportación destacable se refiere a la evaluación colaborativa. En este campo, las tecnologías vienen a asistirnos en relación con la visualización de los procesos colaborativos implicados en una evaluación de estas características. Esta aportación tiene diferentes ilustraciones como los debates virtuales, los foros de conversación y los grupos de trabajo. A continuación se detalla cada una de las aportaciones que se han citado.

\section{Evaluación automática}

Las ventajas de la evaluación automática son tan evidentes como sus limitaciones y ambas realidades se manifiestan en las innumerables aplicaciones de este tipo de evaluación en la red (por ejemplo, MSOR Report, 2003). La mayor ganancia de esta aportación se refleja en la inmediatez de la visualización de la respuesta correcta hecho que es muy importante para los alumnos, pero también para el profesor porque su acción retroalimentativa descansa en ella. La respuesta automática se puede igualar a esa presencia docente en la cual el profesor valida el contenido de lo que el alumno ha contestado. Y al ser un hecho automático que conecta de manera inmediata la pregunta con la validez de la respuesta se trata de una aportación pedagógicamente muy valiosa. También en este tipo de aproximaciones en las que se utiliza una evaluación automática se detecta una programación docente en la que el nivel de aprendizaje es progresivamente más complejo. Por tanto, estamos hablando de una ventaja que se introduce gracias a la tecnología y que en el caso de los contextos virtuales no se ubica en un ordenador sino que se sitúa en la misma red por lo que puede ser compartida.

El evidente inconveniente se refiere a la limitada intercomunicación que se da entre profesor y alumnos, en el sentido que al no ser una comunicación en vivo y ajustada a lo que sucede sino estandarizada, se omite la personalización de las respuestas. También sucede que las premisas sociales de pertenencia a un colectivo de aprendizaje que comparte unos contenidos desembocan en una baja sensación de comunidad virtual. La identidad colectiva es muy importante en los contextos virtuales y no sólo en el marco de la evaluación virtual sino por el mismo hecho de reducir el abandono de estudios de este tipo.

\section{Evaluación enciclopédica}

Las ventajas de las prácticas evaluativas que utilizan este enfoque tienen diferente cariz si se trata de alumnos o de profesores. Por la parte de los alumnos es notorio que consiguen una considerable ganancia mediante un acceso rápido y relativamente cómodo a gran cantidad de información diversa de distintas fuentes en el marco de Internet. Además esta 
información está digitalizada por lo que la elaboración de los documentos evaluativos se puede construir de un modo más sencillo aunque desde luego esta misma realidad supone otros riesgos. Pasando pues a los inconvenientes se considera que este hecho aumenta las posibilidades de plagio creando problemas no sólo instruccionales sino también institucionales (Bates y Fain, 2004). Existen distintas estrategias para que esto no ocurra o para intentar evitarlo al máximo y no sólo de corte sancionador sino también soluciones de tipo pedagógico. Pero lo que se pretende resaltar en este momento siguiendo el objetivo del artículo es que dada la importancia de la gran aportación de información y su potencial nivel de elaboración, el rol del profesor varia sustancialmente dejando de ser ese transmisor que acerca la información a sus alumnos modificando las actuales estrategias de enseñanza.

\section{Evaluación colaborativa}

Se observa que existe un gran número de trabajos y propuestas que se refieren al aprendizaje colaborativo desde los inicios de las prácticas educativas formales en contextos virtuales (CSCL, BSCW, etc.). A pesar de esta presencia la evaluación colaborativa a penas se ha tratado siendo un campo por explorar (Achtemeier, Morris y Finnegan). Como decimos la colaboración educativa virtual se identifica en todas las plataformas de e-learning: foros, debates, hilos de conversación, etc. Lo que también sucede es que estos recursos o bien no entran dentro de la actividad docente principal o bien no se sabe muy bien cómo extraer indicadores para la evaluación del aprendizaje. No existe discusión sobre las ventajas pedagógicas que se desprenden de una acción colaborativa en un contexto virtual adecuadamente guiada en términos sociales y cognitivos al mismo tiempo. Una ventaja metodológica es que la tecnología nos aporta la posibilidad de no sólo evaluar el producto colaborativo sino también el proceso. Este hecho es significativamente diferente del que ocurre en las prácticas presenciales que resulta de mucho interés para una verdadera evaluación formativa.

En el trabajo colaborativo virtual el profesor puede ofrecer y recibir distintos aspectos instruccionales válidos para el seguimiento del aprendizaje. Mediante el planteamiento de grupos virtuales se puede dar soporte individual a los alumnos para llegar a un producto concreto $\mathrm{y}$, por su parte, el profesor tiene la posibilidad de visualizar a distancia, en sus variadas formas, lo que está sucediendo con exactitud en los grupos y quién está aportando cada pieza de trabajo realmente. Relacionando este hecho con la evaluación acreditativa sobre el aprendizaje puede atribuir calificaciones diferentes en función de lo que aporte cada alumno a parte de otorgar una calificación compartida a todo el grupo. Un inconveniente del trabajo virtual en grupo que se sufre con cierta frecuencia es la oposición que marcan ciertos alumnos ante el planteamiento de un trabajo en grupo. Muchos alumnos que optan por una enseñanza en línea tienen la expectativa de realizar las actividades de aprendizaje de manera individual y es precisamente por este factor que escogen esta modalidad formativa sin ataduras aparentes. Quieren llevar a cabo sus estudios a su ritmo, sin tener que ponerse de acuerdo con otros alumnos, especialmente si se trata de una enseñanza básicamente asincrónica. Por tanto, contamos con el peor de los inconvenientes que es la oposición de una parte de los alumnos a realizar trabajos en colaboración aunque ello se manifiesta en unas disciplinas más que en otras, y en unas franjas de edad más que en otras pero, en definitiva, hemos de contar con ello. 
Comentábamos al inicio del artículo la importancia de determinar, en este marco de aportaciones fruto de la introducción de la tecnología en las aulas, los focos de esta evaluación del aprendizaje susceptibles de ser puntos estratégicos de cambio evaluativo. Por lo menos en la evaluación en línea, se apunta, como hemos visto, a que el mayor responsable del cambio son los instrumentos de evaluación. Se ha hablado de los portafolios, de los test, de los trabajos en grupo, etc., puesto que se trata de la parte más vistosa y del mediador más asequible: el instrumento mediante el que se evalúa. Parece que todo ello es lo que preocupa más a los diseñadores instruccionales, a los técnicos que desarrollan la tecnología docente $\mathrm{y}$, en cierto modo, a los propios profesores. Formando parte o no de estos colectivos, en estos momentos estamos en una posición de poder reflexionar sobre esta temática por lo que trasladaríamos el énfasis que se imprime a los instrumentos de evaluación a otros elementos de proceso evaluativo. Creemos que debemos poner ahora el acento en los procesos más psicoeducativos involucrados en la evaluación del aprendizaje. Con ello se propone recuperar temáticas constitutivamente importantes, más allá de los instrumentos de evaluación, como la retroalimentación y el modo como se lleva a cabo en los contextos virtuales.

\section{Proceso de evaluación}

Si se revisa con detalle el proceso completo de evaluación y los componentes técnicos que están en juego vemos que la evaluación es más que los instrumentos de recogida de evidencias evaluativas. Parece que nos ha interesado confundir la evaluación con los instrumentos mediante los que se recogen los datos evaluables y también confundirla con la calificación que merecen dichos aprendizajes. Estos dos hechos se reproducen una y otra vez conformando dos de los mayores mitos con que contamos en el ámbito de la evaluación, la preponderancia de los instrumentos y la tendencia hacia la evaluación acreditativa. Como se observa, los instrumentos responden al objetivo de recoger la información pero es que hay muchos otros retos por lo que se ha de abordar la evaluación desde una visión compleja, no sólo desde la mera fase de recogida de datos. Por lo tanto, necesitaremos otros muchos factores que no desarrollaremos aquí como son: los criterios de evaluación que nos indican la bondad de la evaluación, el acto de emisión de juicios de valor, los objetivos y referentes de evaluación, la conformación de decisiones educativas de mejora conceptual y procedimental comprensibles y justas para profesores y los alumnos, entre otros. Todos estos factores no son en absoluto banales y precisan una configuración muy bien tramada para conseguir cambios sustanciales en las prácticas evaluativas. En este sentido, de todos los elementos citados, uno de los más potentes es el que se ubica en la parte postevaluativa de comunicación de los resultados a los alumnos (Coll et al., 1995). Naturalmente se evalúa para comunicar resultados de diferente naturaleza, cualitativos y cuantitativos, y todo ello contribuye a situar al alumno en la norma general de consecución de un conocimiento concreto y para mejorar progresivamente sus consecuciones. Así apuntábamos que la comunicación de resultados sirve para calificar y ejercer la función más normativa de la evaluación pero también para la desarrollar la más psicopedagógica dando una importancia nuclear a las ayudas educativas y al feedback en un contexto virtual.

\section{Agenda: feedback virtual como derecho y como deber}

La propuesta se concreta en el trasladar la atención del lugar donde la tenemos centrada en estos momentos (en los instrumentos) a la reflexión sobre el diálogo evaluativo que 
se genera a partir de la aplicación de los instrumentos. En concreto, reflexionar sobre el feedback, sobre el feedback virtual y, necesariamente, sobre el aprovechamiento de los contenidos que aporta este feedback virtual al alumno. Creemos que los alumnos tienen derecho a la mejora de sus propias producciones desde el propio diseño de la evaluación y ello también comporta unos deberes para ellos. El feedback virtual abre otros muchos campos de necesaria revisión y llama la atención a los alumnos sobre la calidad de sus aportaciones; por ejemplo, les informa de las diferencias que existen entre participar e interactuar (Moore y Kearsley, 1996). La participación se mide normalmente mediante la cantidad de mensajes que se emiten en un espacio virtual, pero asaltan preguntas razonables a cerca de estas participaciones: ¿cómo son?, ¿se retoman o se ignoran?, ¿influyen en otros alumnos?, etc. En consecuencia, la interacción sería lo que constituye un cambio para ir a cotas cognitivas y sociales más complejas mientras que participar supone simplemente intervenir en un espacio virtual no favoreciendo una influencia dirigida a un cambio cognitivo o provocando una reacción virtual conjunta $y$ compartida.

Existen muchos sistemas virtuales que lo que tienen en cuenta es la participación y su traducción en frecuencia de intervenciones pero no la interacción o la calidad de dicha interacción. Creemos que la clave es la naturaleza de la interacción puesto que involucra a profesores y alumnos de igual manera aunque desde diferentes perspectivas. Como anunciábamos, uno de los núcleos básicos de la interacción es la dinámica establecida mediante los procesos de feedback que adaptan y readaptan de manera progresiva el conocimiento ajustándolo de un modo correcto. Pero no nos podemos quedar sólo en uno de los sentidos de la dirección del feedback, en este caso el iniciado por el profesor hacia el alumno para que potencialmente, si utiliza sus ayudas y consejos, mejore su aprendizaje. En un sistema de evaluación completo hemos de aspirar a incluir el otro sentido de la dirección del feedback, el que va del alumno al profesor en respuesta al aprovechamiento de sus ayudas evaluativas. Es decir, sería preciso completar el diálogo evaluativo y el mismo proceso de enseñanza y aprendizaje conociendo si se aprovecha el feedback virtual y qué modificaciones incluye en la versión original de los primeros aprendizajes. ¿Por qué en los sistemas de evaluación no se incluye algo tan fundamental como el nivel de aprovechamiento del feedback del profesor? ¿Para qué lo emite entonces? ¿Realmente aprovechan los alumnos esas ayudas? ¿Cómo? ¿Qué repercusiones tiene este hecho en su aprendizaje concreto? ¿Se diseñan materiales de estudio y planes docentes de manera que puedan ellos aprovechar ese feedback y puedan realmente incluir mejoras en sus primeras aportaciones? Porque diseñar contextos de enseñanza y aprendizaje virtual significa dotarse de un sistema de evaluación complejo que incluya criterios, juicios, decisiones educativas, retroalimentación, en el marco de un aprovechamiento de las ayudas del profesor para desarrollar un aprendizaje progresivamente más sólido y complejo (Barberà, 2005). En este contexto de mejora progresiva se han de planificar los diseños y las interacciones que traduzcan la evaluación como un ente realmente complejo y articulado que procure una evaluación desde las cuatro perspectivas que apuntábamos: evaluación del aprendizaje, evaluación para el aprendizaje, evaluación como aprendizaje y evaluación desde el aprendizaje.

Presentación del artículo: 05 de julio de 2005

Fecha de aprobación: 30 de julio de 2005

Fecha de publicación de la primera edición: 30 de septiembre de 2005 
Barberà, E. (2016). Aportaciones de la tecnología a la e-Evaluación. RED. Revista de Educación a Distancia. 50(4). Consultado el (dd/mm/aaaa) en http://www.um.es/ead/red/50

\section{Referencias}

Achtemeier, S. D., Morris, L.V. y Finnegan, C.L.(2003) Considerations for developing evaluations of online courses. Journal of Asinchronous Learning Network, 7 (1) Disponible en:

http://www.sloan-c.org/publications/jaln/v7n1/pdf/v7n1_achtemeier.pdf [último acceso $15 / 6 / 2006]$.

Australian National Training Authority (2002). Assessment and Online Teaching. Disponible en: http://pre2005.flexiblelearning.net.au/guides/assessment.pdf [último acceso $12 / 6 / 2006]$.

Barberà, E. (2005). La evaluación de competencias complejas. EDUCERE, 31, 497-504.

Barberà, E. (2003). Estado y tendencias de la evaluación en la educación superior. Boletín REDU, 3 (2), 94-99.

Barrón, H. S. (2004) Seis problemas de los sistemas universitarios de educación en Línea RED, Revista de Educación a Distancia, 12. Disponible en: http://www.um.es/ead/red/12/barron.pdf [último acceso 10/6/2006].

Bates, P. and Fain, M. (2004). Cheating 101: Internet paper mills. Disponible en: http/www.coastal.edu/library/pubs/mills2.html [último acceso 15/6/2006]

Coll, C. (coord.). La evaluación de los aprendizajes en las prácticas evaluativas escolares. Proyecto de Investigación Ministerio de Educación y Ciencia PB 95-1032-1995.

Kati Mäkitalo, Piritta Salo, Päivi Häkkinen y Sanna Järvelä (2001). Analysing the mechanism of common ground in collaborative web-based interaction. Disponible en: http://www.ll.unimaas.nl/euro-cscl/Papers/107.doc [último acceso 15/6/2006].

Moore, M.G. y Kearsley, G. (1996). Distance Education: A system view. Belmont, Wadsworth Publising Company.

MSOR Workshop Reports (2003). Report prepared by J. Morgan, University of Reading. Workshop on the Mathletics online objective tests, Brunel, 8 May 2003. Disponible en:

http://mathstore.ac.uk/newsletter/aug2003/56\%20workshops.pdf [último acceso 15/6/2006]. 